\title{
SPECTRUM OF GALACTIC BACKGROUND RADIATION
}

\author{
J. R. SHAK ESHAFT \\ Mullard Radio Astronomy Observatory, Cambridge, United Kingdom
}

\begin{abstract}
New measurements of the galactic background radiation between 151 and $1407 \mathrm{MHz}$ have been made by Sironi and by Webster. The radio spectrum shows a bend at $\sim 315 \mathrm{MHz}$ in Bridle's Region I and $\sim 100 \mathrm{MHz}$ in Region II, implying magnetic fields of $7.6 \mu \mathrm{G}$ and $2.4 \mu \mathrm{G}$, respectively.
\end{abstract}

(The following summary was prepared from the tapes by the Editors.)

It is well known that studies of the galactic radio background spectrum provide evidence on the magnetic field and cosmic ray electrons in the Galaxy, and over the past $10-12$ yr there have been various attempts to measure the radio spectrum more precisely. These have gone along with measurements of the cosmic-ray-electron spectrum to study the fit between the two spectra in terms of standard synchrotron theory.

The radio measurements must be made using scaled aerials, having the same beams at different wavelengths, to avoid side-lobe problems, for example the observations of Turtle, Bridle and Howell at various frequencies. The only exception is the work of Wielebinski and others using lunar occulations, the assumption being that the Moon is essentially the same size at all wavelengths.

This paper reports new measurements by Sironi (1974), using similar arrays at 151 and $408 \mathrm{MHz}$, and by Webster (1974) at 408,610 , and $1407 \mathrm{MHz}$ with $15^{\circ}$ horns. These beams are much larger than would be desirable, but the scaled-antenna method cannot give a very high resolution all the way from 17.5 to $1407 \mathrm{MHz}$.

The method of interpretation of measurements of this kind is usually in terms of 'a $T-T$ plot,' in which the temperature of a given area at one frequency is plotted against that at another frequency, with the slope giving the temperature spectral index, $\beta$. In this way, any constant background components are eliminated, but it does mean that variations over an area are smoothed out to some extent.

In the time available, we will concentrate on the results for Bridle's 'Regions I and II,' which correspond essentially to the local spiral arm and an interarm region. Plotting temperatures at 408 and $610 \mathrm{MHz}$ against each other for different right ascensions, we get reasonably good straight lines, but there are variations. One such distortion is associated with the North Polar Spur. The temperatures are higher for 408 than for $1407 \mathrm{MHz}$, implying that the Spur has a steeper spectral index between these two frequencies than does the average radiation.

Plotting the results at all available frequencies for the two regions, we obtain very smooth curves, but the two curves are not the same. We can now make use of the observed cosmic-ray electron spectrum for the differential number of electrons with given energy against the energy; this has a slope of -1.8 below an energy of about $3 \mathrm{GeV}$ and -2.6 above $3 \mathrm{GeV}$, although there are some disagreements about details. 
The observed radio spectrum shows a bend at around $315 \mathrm{MHz}$ for Region I and $100 \mathrm{MHz}$ for Region II. Applying the standard synchrotron formula, we can relate the frequency of the bend to the magnetic field and the critical energy, leading to a field of $7.6 \mu \mathrm{G}$ for Region I and $2.4 \mu \mathrm{G}$ for Region II.

\section{References}

Sironi, G.: 1974, Monthly Notices Roy. Astron. Soc. 166, 345.

Webster, A. S.: 1974, Monthly Notices Roy. Astron. Soc. 166, 355.

J. R. Shakeshaft

Mullard Radio Astronomy Observatory,

Cavendish Laboratory,

Free School Lane,

Cambridge CB2 3RQ, United Kingdom

\section{DISCUSSION}

Price: What is the latitude range of the regions considered in this study? Shakeshaft: They cover $40^{\circ}$ or $50^{\circ}$ in galactic latitude. 